\title{
Dynamic Transition in the Structure of an Energetic Crystal during Chemical Reactions at Shock Front Prior to Detonation
}

\author{
Ken-ichi Nomura, Rajiv K. Kalia, Aiichiro Nakano, and Priya Vashishta \\ Collaboratory for Advanced Computing and Simulations, Departments of Chemical Engineering \& Materials Science, \\ Physics \& Astronomy, Computer Science, University of Southern California, Los Angeles, California 90089-0242, USA
}

Adri C. T. van Duin and William A. Goddard III

Materials and Process Simulation Center, Beckman Institute (139-74), California Institute of Technology, Pasadena, California 91125, USA

(Received 26 April 2007; published 5 October 2007)

\begin{abstract}
Mechanical stimuli in energetic materials initiate chemical reactions at shock fronts prior to detonation. Shock sensitivity measurements provide widely varying results, and quantum-mechanical calculations are unable to handle systems large enough to describe shock structure. Recent developments in reactive forcefield molecular dynamics (REAXFF-MD) combined with advances in parallel computing have paved the way to accurately simulate reaction pathways along with the structure of shock fronts. Our multimillionatom REAXFF-MD simulations of 1,3,5-trinitro-1,3,5-triazine (RDX) reveal that detonation is preceded by a transition from a diffuse shock front with well-ordered molecular dipoles behind it to a disordered dipole distribution behind a sharp front.
\end{abstract}

DOI: 10.1103/PhysRevLett.99.148303

Mechanically enhanced chemistry [1] is observed during indentation [2], friction [3], shock and detonation [4-11] of energetic materials [12-14]. A major unsolved problem in this area of mechanochemistry is how strains trigger chemical reactions that cause detonation. Recently, the role of a mechanical stimulus, such as bond bending, in initiating reaction at a shock front has attracted much attention. Manna et al. have shown on the basis of density functional calculation of a TATB crystal that bond-bending of nitro group in TATB molecule closes the HOMO-LUMO gap and have speculated that reaction may proceed at supersonic speed [15]. Atomistic-level understanding of the structure of shock fronts at the onset of detonation requires multimillion-atom simulations with reaction chemistry under high strain rate deformations.

We have performed a series of molecular dynamics (MD) simulations of planar shock on a slab of an 1,3,5trinitro-1,3,5-triazine (RDX) [16,17] crystal. Each RDX molecule consists of 21 atoms [Fig. 1(a)], and the unit cell of the RDX crystal contains 8 RDX molecules [Fig. 1(b)]. The $x, y$, and $z$ axes are aligned with the [100], [010], and [001] crystallographic orientations, respectively. We apply periodic boundary conditions in all directions after adding $2 \mathrm{~nm}$ of vacuum layers on both sides of the slab along the $x$ axis. We have simulated a system of $N=2322432$ atoms with dimensions $318.48 \times 284.08 \times$ $271.76 \AA^{3}$ and also a system of 145152 atoms to confirm that major results do not change with the system size. Initially, the system is relaxed for 1 ps at $5 \mathrm{~K}$ and then quenched to $0 \mathrm{~K}$.

Simulations reported here are based on a scalable implementation of a reactive force-field (REAXFF) MD $[18,19]$ on massively parallel computers [20]. The REAXFF potential energy function consists of bonding and nonbonding inter-
PACS numbers: 82.40.Fp, 02.70.Ns, 62.50.+p

actions. Bonding interactions comprise coordination energy, two-body stretching, three-body bending and fourbody torsion energy functions. Nonbonding interactions consists of van der Waals and Coulomb energies [21], where charge transfer is included by an electronegativity

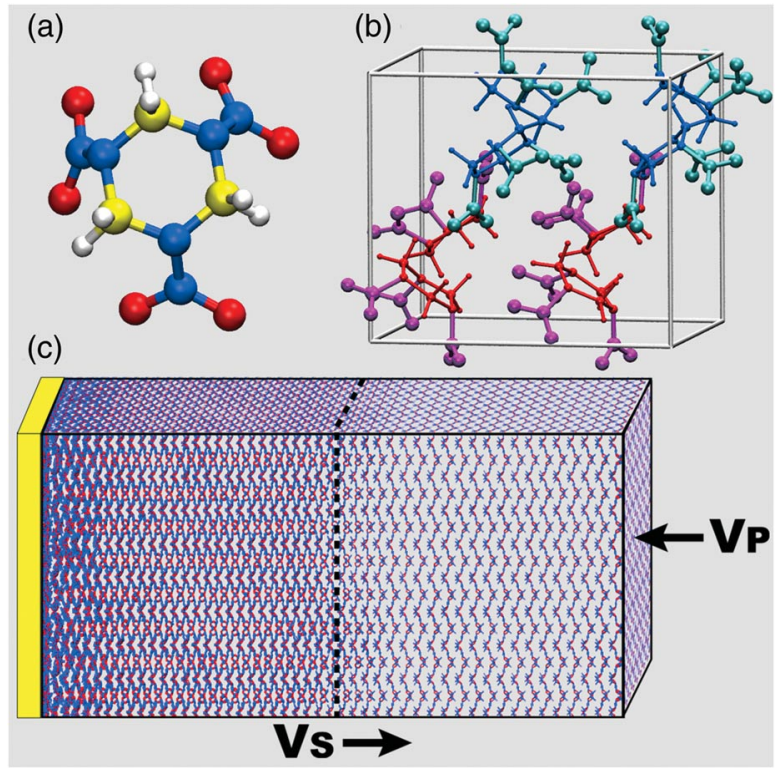

FIG. 1 (color). (a) An RDX molecule with carbon (yellow), hydrogen (white), oxygen (red), and nitrogen (blue) atoms. (b) The unit cell of an RDX crystal contains 8 RDX molecules, which are colored blue and red depending on whether the $\mathrm{NO}_{2}$ groups faces away from (group 1) or faces towards (group 2) the shock plane. The $\mathrm{NO}_{2}$ in group 1 and group 2 molecules are colored in cyan and magenta, respectively. (c) Schematic of a planar shock simulation, where $V_{p}$ and $V_{s}$ are the particle and shock velocities, respectively. 

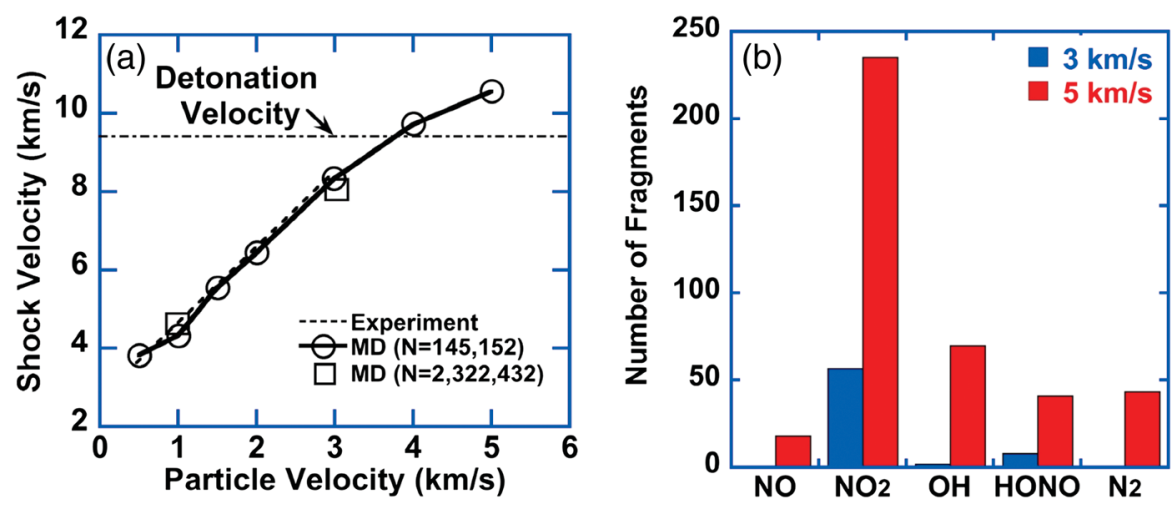

FIG. 2 (color). (a) Molecular dynamics (circles with solid lines to guide the eyes for $N=145,152$ atoms and squares for $N=2,322,432$ atoms) and experimental (dashed) Hugoniot curves (particle velocity $V_{p}$ vs shock velocity $V_{s}$ ) for the RDX crystal. (b) Number of molecular fragments (for $N=145,152$ atoms) produced during 1 ps after shock loading at $V_{p}=3 \mathrm{~km} / \mathrm{s}$ (blue) and $5 \mathrm{~km} / \mathrm{s}$ (red).

equalization scheme [22]. Atomic charges in REAXFF are dynamic variables, which are determined by minimizing the total electrostatic energy at every MD step.

We apply a planar shock parallel to the $y-z$ plane $[23,24]$. The shock is modeled by a momentum mirror (placed in vacuum adjacent to the RDX slab) that reflects the momentum of an atom when it crosses the mirror plane. The RDX slab is initially assigned a translational velocity, $-V_{p}$, in the $x$ direction toward the momentum mirror [Fig. 1(c)]. The particle velocity, $V_{p}$, is varied between 0.5 and $5 \mathrm{~km} / \mathrm{s}$. A shock wave front is identified as a discontinuous drop of the pressure profile [25] along the $x$ direction, and its steady speed after an initial transient defines the shock velocity, $V_{s}$. The shock velocity is obtained from the mass conservation law applied to the shocked and unshocked states.

Figure 2(a) compares the Hugoniot curve $\left(V_{s}\right.$ vs $\left.V_{p}\right)$ obtained from the MD simulation with experimental nonreactive shock data [26]. The figure also shows the experimental detonation velocity, $V_{d}$, i.e., self-sustained reaction wave. Below the detonation velocity, $V_{s}<V_{d}$, the simulation results agree well with the experimental nonreactive Hugoniot data. The MD shock velocity deviates from the linear relation above the detonation velocity. Figure 2(a) also shows that the difference in MD shock velocities between $N=145152$ and 2322432 is less than $6 \%$.

To study chemical reaction pathways, we have performed fragment analysis in which a cluster of covalently-bonded atoms is counted as a molecule. Here, a pair of atoms are considered connected if the bond order of the pair is greater than 0.3 [27]. Figure 2(b) shows the number of molecular products produced in $1 \mathrm{ps}$ after shock loading at $V_{p}=3$ and $5 \mathrm{~km} / \mathrm{s}$, i.e., the shock velocity below and above the detonation velocity [28]. We observe a sudden increase in the number of molecular products above the detonation velocity. At $V_{p}=3 \mathrm{~km} / \mathrm{s}, \mathrm{NO}_{2}$ is found to be the main product, which is also observed in experiments [29]. In contrast, a variety of chemical products such as $\mathrm{OH}, \mathrm{HONO}$, and $\mathrm{N}_{2}$ are obtained at $V_{p}=$ $5 \mathrm{~km} / \mathrm{s}$. A similar trend was observed in an earlier REAXFFMD simulation of a much smaller system [6]. According to a quantum-mechanical calculation of unimolecular decompositions [30], the transition state energy is nearly identical for endothermic $\mathrm{NO}_{2}$ and exothermic $\mathrm{HONO}$ eliminations.

The simulations reveal the coexistence of two qualitatively different molecular responses to shock loading. This can be demonstrated by classifying the molecules into two groups: Group 1, where $\mathrm{NO}_{2}$ molecules face away from the shock front; and group 2, where $\mathrm{NO}_{2}$ molecules face the shock front [Fig. 1(b)]. Figure 3 shows the time variations of the rotational and vibrational temperatures computed from the rotational and vibrational kinetic energies averaged over molecules at $x \sim 2 \mathrm{~nm}$. The rotational energy of each molecule is computed after subtracting the translational energy from the total kinetic energy. The vibrational energy is then obtained by subtracting the rotational energy from the kinetic energy. For group 1 molecules, the rotational motion ensues after the shock arrives and is manifested by a peak in the rotational temperature at $0.8 \mathrm{ps}$; see Fig. 3(a). The rotational energy is subsequently transferred
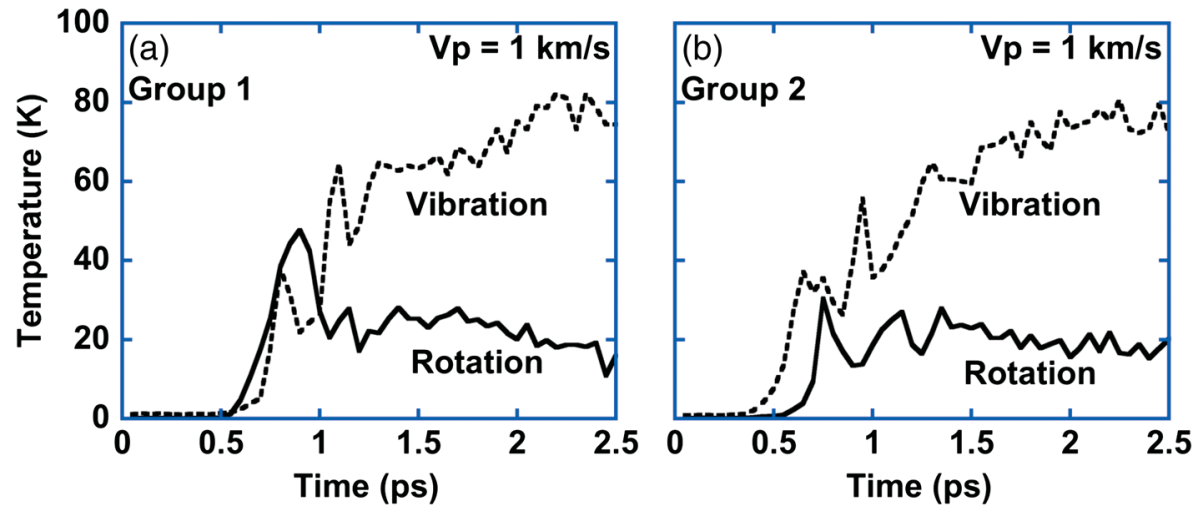

FIG. 3 (color). Time variations of the rotational (solid curves) and vibrational (dashed curves) temperatures of RDX molecules in group 1 (a) and group 2 (b) at $V_{p}=1 \mathrm{~km} / \mathrm{s}$. The repulsive wall starts to interact with the material immediately after the simulation begins at time $t=0$. (a) Group 1 molecules exhibit a transition from rotational to vibrational motion with a 0.3 ps time delay. (b) Group 2 molecules exhibit direct excitation of vibrational modes with the arrival of the shock wave. 
into vibrational modes with a time delay of 0.3 ps. In group 2 molecules, on the other hand, the translational energy is mainly converted into vibrational modes followed by rotational motion shortly after that $[\sim 0.2 \mathrm{ps}$, Fig. 3(b)]. Our simulations thus reveal that the shock response of RDX is bimodal due to different orientations of RDX molecules relative to the shock wave.

We have calculated the dipole moments of the molecules to identify the origin of the bimodal shock response. The dipole moment, $\vec{d}=\sum_{i=l}^{21} q_{i}\left(\vec{r}_{i}-\vec{r}_{\text {com }}\right)$, of an RDX molecule is computed from the charges $\left\{q_{i}\right\}$, atomic positions $\left\{r_{i}\right\}$ in the molecule, and the molecular center-of-mass $\vec{r}_{\text {com. }}$. The top row in Fig. 4 shows one of the molecules in group 1 before and after shock loading with different particle velocities. The magnitude of the dipole is colorcoded and the arrows show the orientation of the dipole moment. The dipole moment is perpendicular to the $\mathrm{C}_{3} \mathrm{~N}_{3}$ ring and pointed towards the ring from the $\mathrm{NO}_{2}$ group. This is due to the fact that oxygen atoms are negatively charged and hydrogen atoms are positively charged. Group 1 molecules respond to the shock by the rotation of the dipole moment as shown in the top row of Fig. 4. The uniaxial strain due to shock loading is initially accommodated by the rigid rotation of the $\mathrm{C}_{3} \mathrm{~N}_{3}$ ring so that it is perpendicular to the shock direction. Subsequent conformational changes due to the high-pressure state in $\mathrm{NO}_{2}$ groups create a closely packed structure.

Group 2 molecules with $\mathrm{NO}_{2}$ groups facing the shock front shrink in the shock direction and elongate in the perpendicular directions by bond bending. This is akin to

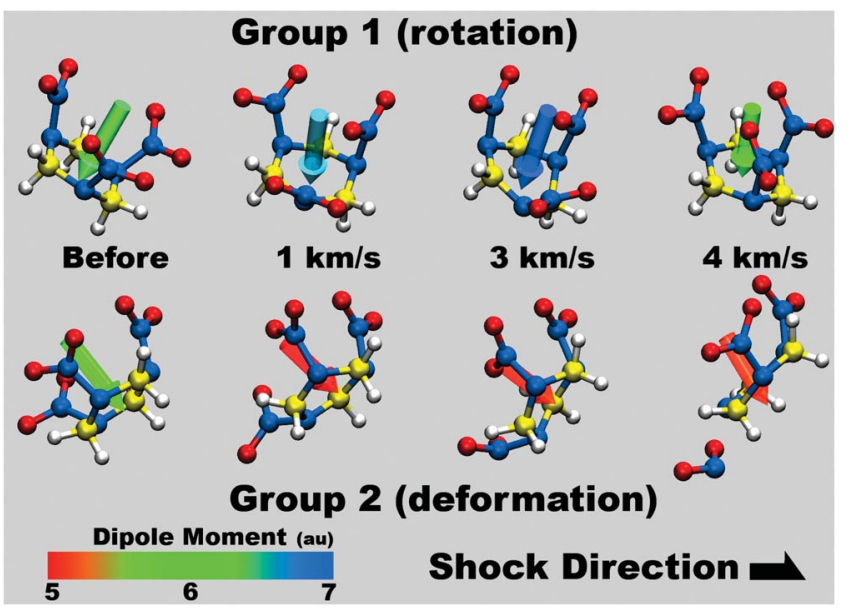

FIG. 4 (color). Snapshots of an RDX molecule from group 1 (top row) and group 2 (bottom row) before shock loading and 1 ps after the arrival of the shock front with particle velocities $V_{p}=1,3$, and $4 \mathrm{~km} / \mathrm{s}$ (from left to right). Yellow, white, red, and blue spheres are carbon, hydrogen, oxygen, and nitrogen atoms, respectively. The arrows indicate the directions of molecular dipole moments and the colors represent the magnitude of the dipole moment. Group 1 molecules rotate so that the $\mathrm{C}_{3} \mathrm{~N}_{3}$ ring is oriented perpendicular to the direction of shock propagation, whereas group 2 molecules deform and are flattened, which reduces the dipole moment. the Poisson effect. The deformation reduces the magnitude of the dipole moment of group 2 molecules steadily from 6 to 5 a.u. as the particle velocity increases (see the bottom row in Fig. 4). It should be noted that group 1 molecules are rather inert to shock loading and reactions are more likely initiated within group 2 molecules, as indicated by the detached $\mathrm{NO}_{2}$ group at $V_{p}=4 \mathrm{~km} / \mathrm{s}$ in the bottom row. This shows that the sensitivity of the molecules in a crystalline energetic material is governed by molecular orientations relative to the direction of shock propagation. Focused laser-induced shock experiments combined with fast laser spectroscopy [31] may be able to detect the predicted bimodal response of RDX molecules.

We have observed the formation of a nanostructure composed of these two types of molecules behind the shock front. Figures 5(a) and 5(b) provide side views of a slice (15 $\AA$ thick) of the RDX crystal under [100] shock loading at $V_{p}=1$ and $3 \mathrm{~km} / \mathrm{s}$, respectively. Here, as in Fig. 4, the arrows represent the dipole moments of RDX molecules. The crystal is a stack of alternating layers consisting of group 1 and group 2 molecules. This is clearly seen behind (left-hand side of) the shock front, where layers of blue (group 1) and red (group 2) arrows are alternately stacked. The shock front, the boundary between the shocked and unshocked regions, is not sharp in the case of weak shock $\left(V_{p}=1 \mathrm{~km} / \mathrm{s}\right)$; see Fig. 5(a). The dipoles, however, are well aligned behind the shock front in Fig. 5(a). In contrast, near the onset of detonation at $V_{p}=3 \mathrm{~km} / \mathrm{s}$, the shock front is sharp and the dipole moment distribution is disordered behind the shock front as shown in Fig. 5(b) [32].

Figures 5(c) and 5(d) show the polar and azimuthal angles of the molecular dipole moment relative to the shock direction for a group 1 molecule at $V_{p}=1$ and $3 \mathrm{~km} / \mathrm{s}$, respectively. At $V_{p}=1 \mathrm{~km} / \mathrm{s}$, the polar angle changes continuously from $120^{\circ}$ to $90^{\circ}$ (perpendicular to the shock) over an interval of $1 \mathrm{ps}$. At $V_{p}=3 \mathrm{~km} / \mathrm{s}$, the polar and azimuthal angles change abruptly at $1.5 \mathrm{ps}$; see Fig. 5(d). The molecular response thus exhibits a crossover from continuous molecular rotations and deformations to discontinuous bond breaking near the detonation velocity.

In conclusion, our multimillion-atom reactive MD simulations reveal bimodal response for an RDX crystal under shock loading. A group of molecules with $\mathrm{NO}_{2}$ group facing away from the shock absorbs the translational energy by undergoing rotation, whereas another group of molecules with $\mathrm{NO}_{2}$ group facing the shock does so by becoming flat. Group 1 molecules are rather inert to shock loading, whereas the N-N bonds in group 2 molecules tend to dissociate due to mechanical deformation [33]. This may explain why more $\mathrm{NO}_{2}$ fragments are found in experiments and simulations of shocked RDX crystal despite the fact that the transition state energy of the N-N bond dissociation is nearly identical to that of HONO elimination in the gas phase. It is noteworthy that the fragment analysis shows a nearly identical ratio between $\mathrm{HONO}$ and $\mathrm{NO}_{2}$ 

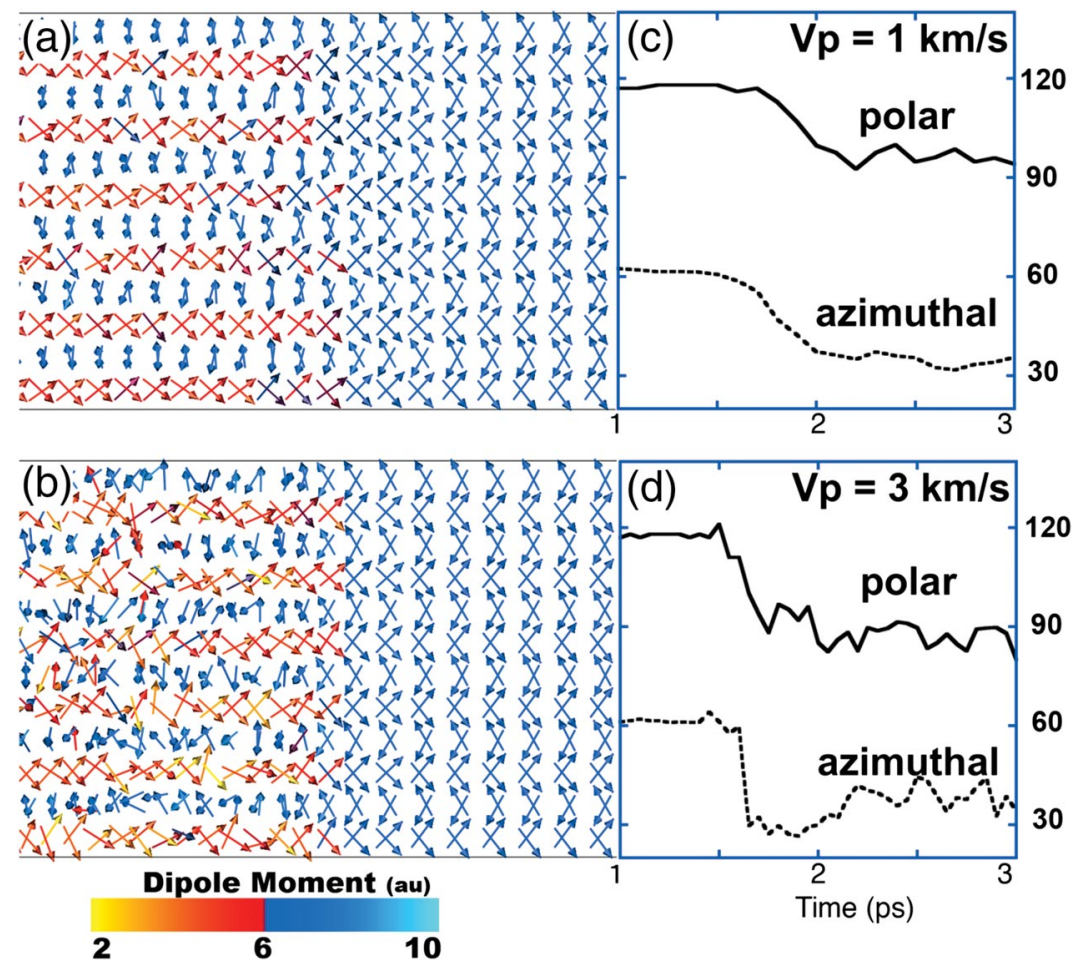

FIG. 5 (color). Molecular dipole moments near the shock front at $V_{p}=$ $1 \mathrm{~km} / \mathrm{s}$ (a) and $3 \mathrm{~km} / \mathrm{s}$ (b). The shock front is diffuse and the dipole distribution is well ordered behind the shock at $V_{p}=1 \mathrm{~km} / \mathrm{s}$ (a). In contrast, a sharp shock front and disordered molecular dipole distribution are observed at $V_{p}=$ $3 \mathrm{~km} / \mathrm{s} \mathrm{(b).} \mathrm{Also} \mathrm{shown} \mathrm{are} \mathrm{the} \mathrm{polar} \mathrm{and}$ azimuthal angles of the dipole moments of a single group 1 molecule at $V_{p}=$ $1 \mathrm{~km} / \mathrm{s}$ (c) and $3 \mathrm{~km} / \mathrm{s}$ (d). Continuous variation of angles at $V_{p}=1 \mathrm{~km} / \mathrm{s}$ becomes abrupt at $V_{p}=3 \mathrm{~km} / \mathrm{s}$. populations, i.e., 0.14 at $V_{p}=3 \mathrm{~km} / \mathrm{s}$ and 0.17 at $5 \mathrm{~km} / \mathrm{s}$. The discrete jumps in the polar and azimuthal angle profiles of the dipole moment at the onset of detonation indicates a crossover mechanism in molecular strain from the nonreactive to the detonation regime.

This work was partially supported by ARO, DTRA, DOE, and NSF. Simulations were performed at DOD Major Shared Resource Centers under a Challenge grant and at the University of Southern California using the 5384-processor Linux cluster at the Research Computing Facility and the 2048-processor Linux clusters at the Collaboratory for Advanced Computing and Simulations.

[1] J. J. Gilman, Science 274, 65 (1996).

[2] J. Sharma et al., Appl. Phys. Lett. 78, 457 (2001).

[3] W. P. King, S. Saxena, and B. A. Nelson, Nano Lett. 6, 2145 (2006).

[4] B. M. Rice et al., Phys. Rev. E 53, 611 (1996).

[5] D. W. Brenner et al., Phys. Rev. Lett. 70, 2174 (1993).

[6] A. Strachan et al., Phys. Rev. Lett. 91, 098301 (2003).

[7] A. Cohen et al., Laser Ignition of Propellants and Explosives (Storming Media, Washington, D.C., 1998).

[8] J. J. Granier, T. Mullen, and M. L. Pantoya, Combust. Sci. Technol. 175, 1929 (2003).

[9] M. M. Hurley et al., Abstr. Pap.-Am. Chem. Soc. 220, U284 (2000)

[10] K. L. McNesby et al., Combust. Flame 142, 413 (2005).

[11] W. H. Wilson, M. P. Kramer, and R. W. Armstrong, Abstr. Pap.-Am. Chem. Soc. 221, U608 (2001).

[12] R. W. Shaw, T. B. Brill, and D. L. Thompson, Overview of Recent Research on Energetic Materials (World Scientific, Singapore, 2005).
[13] V. Yang, T. B. Brill, and W.Z. Ren, Solid Propellant Chemistry, Combustion, and Motor Interior Ballistics (AIAA, Reston, VA, 2000).

[14] R. A. Yetter et al., J. Propul. Power 11, 683 (1995).

[15] M. R. Manaa, Appl. Phys. Lett. 83, 1352 (2003).

[16] T. D. Sewell and C. M. Bennett, J. Appl. Phys. 88, 88 (2000).

[17] D. C. Sorescu, B. M. Rice, and D. L. Thompson, J. Phys. Chem. B 101, 798 (1997).

[18] A. C. T. van Duin et al., J. Phys. Chem. A 105, 9396 (2001).

[19] A.C. T. van Duin et al., J. Phys. Chem. A 107, 3803 (2003).

[20] A. Nakano et al., Comput. Mater. Sci. 38, 642 (2007).

[21] In REAXFF, Coulomb interaction is screened by a taper function with a cutoff radius of $10 \AA$.

[22] W. J. Mortier, S. K. Ghosh, and S. Shankar, J. Am. Chem. Soc. 108, 4315 (1986).

[23] T. C. Germann et al., Phys. Rev. Lett. 84, 5351 (2000).

[24] B. L. Holian, Shock Waves 13, 489 (2004).

[25] P. S. Branicio et al., Phys. Rev. Lett. 96, 065502 (2006).

[26] T. R. Gibbs and A. Popolato, LASL Explosive Property Data (1980).

[27] The bond order cutoff value 0.3 is used in eariler studies of an energetic molecule TATP using the REAXFF, see A. C. T. van Duin et al., J. Am. Chem. Soc. 127, 11053 (2005).

[28] Up to $V_{p}=2 \mathrm{~km} / \mathrm{s}$, no molecular fragment is observed.

[29] F. J. Owens and J. Sharma, J. Appl. Phys. 51, 1494 (1980).

[30] D. Chakraborty et al., J. Phys. Chem. A 104, 2261 (2000).

[31] J. E. Patterson et al., Phys. Rev. Lett. 94, 015501 (2005).

[32] A similarly sharp shock front is observed for $V_{s}>V_{d}$.

[33] Nearly 3 times greater than group 1 molecules for 1 ps. 